\title{
Distributed Optimization for Radar Mission Coordination
}

\author{
Tracie A. Severson and Derek A. Paley
}

\begin{abstract}
This paper presents optimization algorithms that enable multiple ship-based radar systems to maximize their collective target search area while concurrently solving an optimal sensor-to-target assignment problem. We present theoretically justified strategies that determine the optimal ship location and search-area radius for each radar in a given threat environment satisfying a pre-defined resource reserve constraint. We then solve the optimal target-assignment problem by balancing the radar tasking among all participating sensors and show the resulting increase in the number of trackable targets in the combined search area. We provide analytical and numerical simulations to illustrate the algorithm performance.
\end{abstract}

\section{INTRODUCTION}

Modern radar systems perform a variety of tasks, including surveillance, tracking, identification, discrimination, and engagement support. For Integrated Air and Missile Defense missions [1], multi-function radar systems primarily perform surveillance and tracking functions in order to simultaneously protect and defend against multiple long and shortrange threats, most often in a communication-constrained and resource-limited environment. While pre-mission planning can anticipate and allocate initial surveillance responsibilities based on the most recent intelligence data, dynamic sensor tasking and autonomous resource coordination increases the number of threats that are detected, tracked, and engaged. Radar resource coordination also reduces the likelihood of multiple radars tracking the same object, thus freeing up resources for additional targets [2],[1].

Radar resource allocation for multi-functions radars has been considered in previous research. In [3], the authors present an approach to resource allocation that optimizes a single radar's quality of service while searching for and tracking targets subject to resource constraints. For multiple radars, Kang and Lee demonstrated an increase in the number of targets detected when they statically partitioned the combined search coverage area of multiple phased-array radars into angular search regions based on relative load-balancing and expected target arrival [4].

Multi-agent coordination algorithms that consider sensor resource constraints have also been studied for search and track applications [5],[6],[7]. Algorithms that utilize centralized planning nodes or centralized network optimization

This material is based upon work supported by the Office of Naval Research Grant No. N00174-09-2-00023.

T. Severson is a graduate student in the Department of Aerospace Engineering, University of Maryland, College Park, MD 20742, USA tseverso@umd. edu

D. Paley is an assistant professor in the Department of Aerospace Engineering, University of Maryland, College Park, MD 20742, USA dpaley@umd. edu models for multi-vehicle coordination demonstrate quicker search-area coverage and target neutralization than when agents are not coordinated [8].

Since many radar systems employed for search and track of ballistic missile and air threats are deployed on mobile, maritime platforms that communicate via constrained networks, available bandwidth limits the amount of information exchanged between units. Additionally, sensors may dynamically join and/or leave a network due to adverse conditions, such as jamming or environmental constraints. In [9], the authors present a distributed protocol that demonstrates consensus for a connected network by exchanging a single bit of information at each time step. [10] shows that consensus can be reached even in the presence of inconsistent situational awareness, which can occur as a result of network latencies and varying sensor accuracy. Since target locations may not be known a priori, optimization algorithms such as those described in [6],[11], demonstrate detection of a group of distinct target locations within a prescribed environment without assuming exact target coordinates.

Our objective is to apply tools from optimization and control to design theoretically justified methods for dynamic, autonomous radar coordination that maximize the collective radar search area and concurrently optimize the radar-totarget assignment. The technical approach is to optimize each radar's search radius and spacing from other radars in order to maximize the collective search area and ensure a closed search boundary around all ships. We view this approach as distributed because in contrast to a connected network, where all information is shared among each platform and identical calculations are performed to reach an optimal solution, the ship network considered in this paper may be directed and unconnected. Not every coordinating radar receives all available information when solving for the optimal search sector radius and position that maximizes the collective search area. We assign the target tracking responsibilities so that the radar tasking among all ships is as balanced as possible.

The contributions of this paper are (1) we develop a mathematical model of radar systems that concurrently search for and track targets within a specified area subject to a radar resource constraint; (2) we provide an analytical method to maximize the collective search area of multiple coordinated radars while concurrently tracking a prescribed number of targets within that area; and (3) we provide an optimal targetto-sensor assignment for multiple coordinated radars.

The outline for this paper is as follows. Section II describes an idealized model of a multi-function radar system. Section III presents the theoretical results for maximizing the 


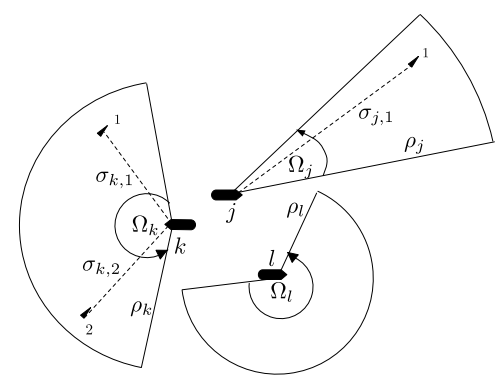

Fig. 1: Radar resources for ship $k$ are consumed based on the range $\sigma_{k, m}$ to target $m$ and the dimensions $\left(\rho_{k}, \Omega_{k}\right)$ of the search sector.

collective search area and numerical simulations that validate and extend the results to an arbitrary number of participating radars. Section IV describes the target-assignment algorithm using simulations to illustrate various operational scenarios. Section V summarizes the paper and our ongoing research.

\section{RADAR SYSTEM MODEL}

In a multi-function radar system, the radar performs two primary tasks - search and track [12]. During search, the radar sends out a focused beam covering a subset of the search sector and looks for a target. If no target is detected, then the search beam moves to the next location until the entire sector is searched. If a target is detected, the radar tracks it by periodically sampling the projected target location. If resources are fully consumed by tracking targets, the radar will suspend searching for new targets until resources are freed from tracking requirements, since tracking current targets takes priority over searching for new ones.

We study the radar tasking of $N$ ships with identical radars in which some or all of the ships are able to coordinate their search and track tasks over a communication network. The operating environment is the set of all possible ship positions $(x, y) \in \mathbb{R}^{2}$ and target locations. The distance between ship $k$ and target $m$ is denoted $\sigma_{k, m}=\sqrt{\left(x_{k}-x_{m}\right)^{2}+\left(y_{k}-y_{m}\right)^{2}}$. Likewise, the distance between ship $k$ and ship $j$ is denoted $\sigma_{k, j}=\sqrt{\left(x_{k}-x_{j}\right)^{2}+\left(y_{k}-y_{j}\right)^{2}}$, where $j, k \in\{1, . ., N\}$.

We assume that surveillance tasks consume radar resources as a linear function of both the angular search sector $\Omega$ and radius $\rho$ of the search sector, whereas tracking tasks consume resources as a linear function of the range $\sigma$ to the target (see Fig. 1). In ongoing research we are extending the model to incorporate a three-dimensional nonlinear radar [13],[12]. We also specify a resource reserve, $\varepsilon \geq 0$, which represents the fraction of the total (unit) resource that cannot be used for search or track. Coordinated ships can track targets in another ship's search area; an uncoordinated ship can only track targets within its own search area.

The models for resource consumption for search, $S_{k}=$ $S\left(\rho_{k}, \Omega_{k}\right)$, and track, $T_{k, m}=T\left(\sigma_{k, m}\right)$, by ship k are

$$
\begin{gathered}
S_{k}=\mathrm{a} \rho_{k}+\mathrm{b} \Omega_{k}, \\
T_{k, m}=\mathrm{c} \sigma_{k, m}
\end{gathered}
$$

where a, b, c $\in \mathbb{R}_{+}$are radar parameters and $m \in\{1, . . M\}$ is the target index. Every ship consumes resources to meet its search requirements, but when ships coordinate on target assignment, only the ship assigned to track the target of interest consumes resources for tracking. The total resource consumed by ship $k$ is

$$
P_{k}=S_{k}+\sum_{j=1}^{M} T_{k, m}
$$

where $M$ is the numbers of targets tracked by ship $k$. The resource reserve constraint is

$$
P_{k} \leq 1-\varepsilon
$$

where $\varepsilon$ is the prescribed resource reserve. Equations (1)-(4) represent an idealized two-dimensional model of the full set of radar range equations that represent the resource usage for a ship searching and tracking targets.

\section{SEARCH AREA MAXIMIZATION}

An optimal solution to the search-area problem maximizes the combined search area of $N$ ships that can be maintained even after initialization of one or more target tracks. In this section we assume targets are located at the search area boundary since this will consume the greatest amount of resources. We formulate the optimization problem as follows:

$$
\max _{x} f(x) \text { s.t. } g(x) \leq 0,
$$

where $x$ is the set of variables to be optimized, $f(x)$ is the objection function, and $g(x) \leq 0$ is the constraint. We illustrate the procedure for one, two and three-ship scenarios and use numerical simulations to illustrate the results.

\section{A. Single Ship}

For $N=1, x=\rho$ is the radius of a circular search sector and $f(\rho)=\pi \rho^{2}$ is the area. The constraint $g(\rho)$ represents the resource reserve constraint (4):

$$
\begin{aligned}
g(\rho) & =M T(\rho)+S(\rho, 2 \pi)+\varepsilon-1 \\
& =\alpha \rho+\beta \leq 0
\end{aligned}
$$

where

$$
\alpha=a+M c \text {, and } \beta=2 \pi b-1+\varepsilon \leq 0 .
$$

We have the following result.

Lemma 1: Let $\rho$ be the search-sector radius of a single ship capable of tracking up to $M$ targets. If $\Omega=2 \pi$, the largest radius $\rho^{*}$ that can be maintained for the resource reserve $\varepsilon$ is $\rho^{*}=-\beta / \alpha$, where $\alpha$ and $\beta$ are given by (7).

Proof: Since $f(\rho)$ monotonically increases with $\rho$ and $\alpha>0$, then $\rho^{*}$ satisfies $g\left(\rho^{*}\right)=0$. 


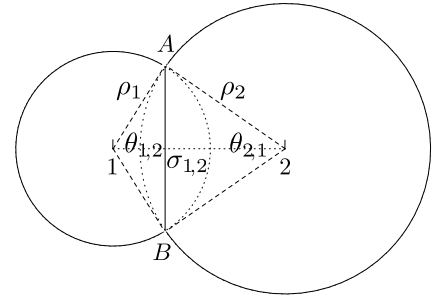

Fig. 2: Combined search area for two ships

\section{B. Two Ships}

For $N=2, x=\left(\rho_{1}, \rho_{2}, \sigma\right)$ represents the search-area radius $\rho_{1}$ of ship $k=1$, the search-area radius $\rho_{2}$ of ship $k=2$, and the distance $\sigma=\sigma_{1,2}$ between the ships. First we consider the case $\rho_{1}=\rho_{2}=\rho$ for which $f\left(\rho_{1}, \rho_{2}, \sigma_{12}\right)=f(\rho, \sigma)$ is the combined search area shown inside the solid lines of Fig. 2. (If the search sectors do not overlap, then $\rho_{1}=\rho_{2}=$ $\rho^{*}$ given by Lemma 1.) To calculate the total area, we add the individual search areas and subtract the area of overlap. The area of overlap is found by calculating the area of each sector described by $\theta_{1,2}=\theta_{2,1}=\theta$ and subtracting from this the area of the triangles $1 \mathrm{AB}$ and $2 \mathrm{AB}$. Using the identity $\cos u \sin v=(\sin (u+v)+\sin (u-v)) / 2$, we find

$$
\begin{aligned}
f(\rho, \sigma) & =2 \pi \rho^{2}-2\left(\frac{\theta}{2 \pi} \pi \rho^{2}\right)+4\left(\frac{1}{2} \rho \cos \frac{\theta}{2} \rho \sin \frac{\theta}{2}\right) \\
& =(2 \pi-\theta+\sin \theta) \rho^{2} .
\end{aligned}
$$

We express $\theta$ in terms of $\sigma$ and $\rho$ by solving $\cos (\theta / 2)=$ $\sigma /(2 \rho)$ for $\theta$. Since $1-\sigma /(2 \rho)>0$ in order for the sensor areas to overlap, this implies that $\sigma /(2 \rho)<1$ and therefore $0<\cos (\theta / 2)<1$. Note that $\sin \theta-\theta \leq 0$ decreases as $\theta$ increases. Thus the total search area is maximized when $\sin \theta-\theta$ is maximized, which corresponds to smaller values of $\theta$. We perform a Taylor series expansion of $\cos (\theta / 2)$ about $\theta / 2=0$ to obtain

$$
\frac{\sigma}{2 \rho}=\cos \left(\frac{\theta}{2}\right)=1-\frac{\theta^{2}}{8}+\text { H.O.T. }
$$

Dropping the higher order terms and solving for $\theta$ yields

$$
\theta \approx 2 \sqrt{2-\frac{\sigma}{\rho}} .
$$

The resource-reserve constraint (4) reflects the observation that when two ships are coordinating, the area of overlap need not be searched in order to maintain a closed search boundary. The constraint equation is

$$
\begin{aligned}
g(\rho, \sigma) & =\alpha \rho+b(2 \pi-\theta)-1+\varepsilon \\
& =\alpha \rho+\beta-b \theta \leq 0,
\end{aligned}
$$

where $\alpha$ and $\beta$ are as in (7). We have the following result.

Lemma 2: Let $\rho$ be the search sector radius of two identical ships separated by $\sigma<2 \rho$ and each capable of tracking up to $M$ targets. The maximum search radius $\rho^{*}$ that

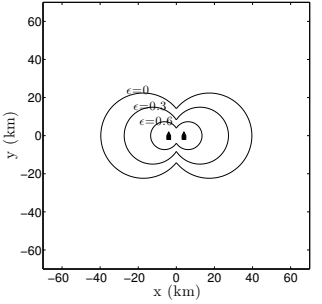

(a) $N=2$, Coordinated

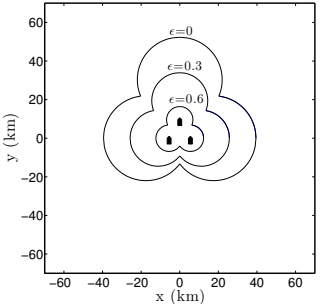

(b) $N=3$, Coordinated
Fig. 3: Search volume maximization: The search volume is a function of the reserve limit and the network topology.

can be maintained for the resource reserve $\varepsilon$ is the largest root of

$$
a_{4} \rho^{3}+a_{3} \rho^{2}+a_{2} \rho+a_{1}=0,
$$

where $a_{4}=-5 \alpha^{3} /\left(6 b^{3}\right), a_{3}=-2 \alpha^{2} \beta /\left(b^{3}\right), a_{2}=$ $\left(-3 \alpha \beta^{2}\right) /\left(2 b^{3}\right)$, and $a_{1}=4 \pi-\beta^{3} /\left(3 b^{3}\right)$; the optimal ship separation is

$$
\sigma^{*} \approx \rho^{*}\left(2-\left(\frac{\alpha \rho^{*}+\beta}{2 b}\right)^{2}\right) .
$$

Fig. 3a illustrates the results of the $N=2, M=1$ searcharea maximization for $\varepsilon=0,0.3$, and 0.6 .

Next we consider a scenario in which the search radii $\rho_{1}$ and $\rho_{2}$ are not equal. Specifically, we consider the case when $\rho_{1}=\rho^{*}$ (the $N=1$ optimal radius), and optimize $\rho_{2}$ and $\sigma$ to maximize the combined search area. This scenario demonstrates the interoperability of the proposed algorithm with pre-existing uncoordinated radar tasking. The objective function $f\left(\rho_{1}, \rho_{2}, \sigma\right)$ is the combined search area and $x=$ $\left(\rho_{1}, \rho_{2}, \sigma\right)=\left(\rho^{*}, \rho_{2}, \sigma\right)$. We have

$f\left(\rho^{*}, \rho_{2}, \sigma\right)=\frac{\rho^{* 2}}{2}\left(2 \pi-\theta_{1,2}+\sin \theta_{1,2}\right)+\frac{\rho_{2}^{2}}{2}\left(2 \pi-\theta_{2,1}+\sin \theta_{2,1}\right)$

where

$$
\begin{aligned}
& \theta_{1,2}=2 \arccos \left(\frac{\sigma^{2}+\rho^{* 2}-\rho_{2}^{2}}{2 \sigma \rho^{*}}\right) \\
& \theta_{2,1}=2 \arccos \left(\frac{\sigma^{2}-\rho^{* 2}+\rho_{2}^{2}}{2 \sigma \rho_{2}}\right) .
\end{aligned}
$$

Our objective function is maximized when both $\theta_{1,2}$ and $\theta_{2,1}$ are small and we express $\theta_{1,2}$ and $\theta_{2,1}$ in terms of $\sigma$ and $\rho_{2}$ using Taylor series expansions:

$$
\begin{aligned}
& \frac{\sigma^{2}+\rho^{* 2}-\rho_{2}^{2}}{2 \sigma \rho^{*}} \approx \cos \left(\frac{\theta_{1,2}}{2}\right)=1-\frac{\theta_{1,2}^{2}}{8} \\
& \frac{\sigma^{2}-\rho^{* 2}+\rho_{2}^{2}}{2 \sigma \rho_{2}} \approx \cos \left(\frac{\theta_{2,1}}{2}\right)=1-\frac{\theta_{2,1}^{2}}{8}
\end{aligned}
$$

Solving for $\theta_{1,2}$ and $\theta_{2,1}$ yields

$$
\begin{aligned}
\theta_{1,2} & \approx 2 \sqrt{2\left(1-\frac{\sigma^{2}+\rho^{* 2}-\rho_{2}^{2}}{2 \sigma \rho^{*}}\right)} \\
\theta_{2,1} & \approx 2 \sqrt{2\left(1-\frac{\sigma^{2}-\rho^{* 2}+\rho_{2}^{2}}{2 \sigma \rho_{2}}\right)} .
\end{aligned}
$$




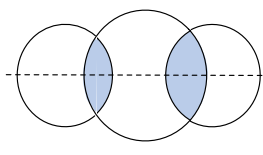

Scenario 1

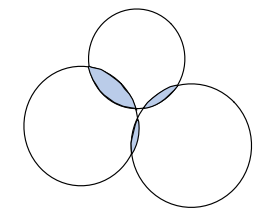

Scenario 2

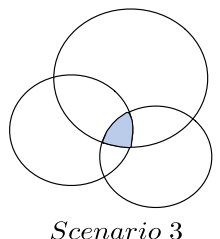

Scenario 3
Fig. 4: Three possible scenarios for $N=3$ sensors

We find the distance $\sigma$ between the two ships as a function of the search radius $\rho_{2}$ and search angle $\theta_{2,1}$ by solving (20) for $\sigma$ :

$$
\sigma^{2}+\sigma\left(\frac{\rho_{2} \theta_{2,1}^{2}}{4}-2 \rho_{2}\right)+\rho_{2}^{2}-\rho^{* 2} \approx 0
$$

Equation (21) yields two positive real roots with one root less than one and the other greater than one. We choose the larger root in order to maximize (14) and solve (11) with $\rho$ replaced by $\rho_{2}$ and $\theta$ replaced by $\theta_{2,1}$ to obtain

$$
\theta_{2,1}=\frac{\alpha \rho_{2}+\beta}{b} \text {. }
$$

Substituting (22) into (21) yields

$$
\begin{aligned}
\sigma & =\frac{4}{8 b^{2}} \sqrt{a_{5} \rho_{2}^{6}+a_{4} \rho_{2}^{5}+a_{3} \rho_{2}^{4}+a_{2} \rho_{2}^{3}+a_{1} \rho_{2}^{2}+4 b^{4} \rho^{* 2}} \\
& -\frac{1}{8 b^{2}}\left(\alpha^{2} \rho_{2}^{3}+2 \alpha \beta \rho_{2}^{2}+\left(\beta^{2}-8 b^{2}\right) \rho_{2}\right)
\end{aligned}
$$

where $a_{5}=\alpha^{4} / 16, a_{4}=\beta \alpha^{3} / 4, a_{3}=\left(3 \beta^{2} \alpha^{2}-\right.$ $\left.8 \alpha^{2} b^{2}\right) / 8, a_{2}=\left(\beta^{3} \alpha-2 \beta \alpha b^{2}\right) / 4$, and $a_{1}=\left(\beta^{4}-\right.$ $\left.16 \beta^{2} b^{2}\right) / 16$.

Using $\sin \theta_{1,2} \approx \theta_{1,2}$ and $\sin \theta_{2,1} \approx \theta_{2,1}$ in (14) yields

$$
f\left(\rho^{*}, \rho_{2}, \sigma\right) \approx \pi \rho^{* 2}-\frac{\theta_{1,2}^{3} \rho^{* 2}}{12}+\pi \rho_{2}^{2}-\frac{\theta_{2,1}^{3} \rho_{2}^{2}}{12} .
$$

Taking the derivative of (24) with respect to $\rho_{2}$ we have

$\frac{\partial f}{\partial \rho_{2}}=-\frac{1}{4} \rho^{* 2} \theta_{1,2}^{2} \frac{\partial \theta_{1,2}}{\partial \rho_{2}}+2 \pi \rho_{2}-\frac{1}{6} \theta_{2,1}^{3} \rho_{2}-\frac{1}{4} \rho_{2}^{2} \theta_{2,1}^{2} \frac{\partial \theta_{2,1}}{\partial \rho_{2}}$,

where $\theta_{2,1}$ is given by (22) with $\partial \theta_{2,1} / \partial \rho_{2}=\alpha / b$; and $\theta_{1,2}$ is given in (19). We evaluate $\partial f / \partial \rho_{2}=0$ to yield the optimal search radius $\rho_{2}^{*}$, and use our results in (24) to solve for the optimal spacing, $\sigma^{*}$. (Results omitted for brevity.)

\section{Three or More Ships}

Here we consider three or more ships with multi-function radars that are tasked to protect a prioritized list of defended assets and/or search specific launch areas for targets. It is unlikely for $N>3$ ships to have overlapping search areas due to the large spacing between the target launch locations as compared to the sensor search area. By solving the $N=1,2$, and 3 search-area maximization problems, we have effectively solved it for an arbitrary number of ships, assuming that no more than three ships have overlapping search areas.

The $N=3$ analysis seeks to maximize the combined
TABLE I: Search-Area Maximization Algorithm, $N=3$

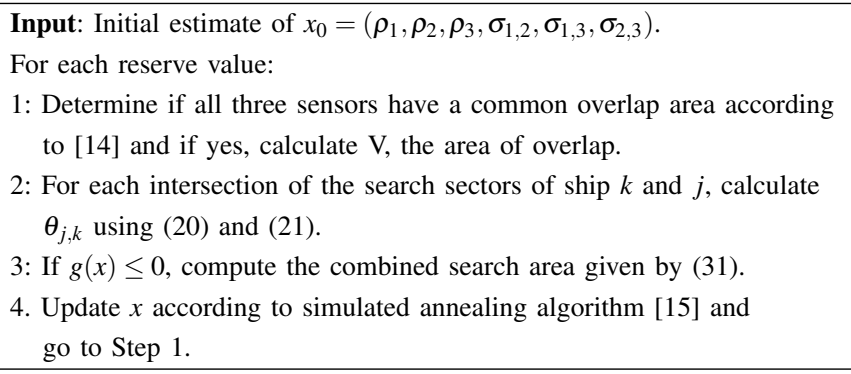

search area $f(x)$, subject to the reserve constraint $g(x)$, where $x=\left(\rho_{1}, \rho_{2}, \rho_{3}, \sigma_{1,2}, \sigma_{1,3}, \sigma_{2,3}\right)$. Note $f(x)$ is the total area searched by all three sensors less any overlap area.

We consider three possible scenarios for $N=3$ ships, depending on the desired search mission (see Fig. 4). Scenario 1 occurs when the centers of the individual search areas are collinear, e.g., the ships form a search fence to detect targets arriving from the same direction or for searching along a border or coastline; Scenario 2 occurs when the objective is to provide a screen against targets that can originate from any direction and can be detected, tracked, and engaged before they reach the center of the search area. Scenario 3 is a special case of Scenario 2 and occurs when all three search areas have a common overlap area, e.g., if the search area is protecting a high value asset located in the search area center and the sensors require the ability to detect and track throughout the entire search area. In this scenario, the area of common overlap is called a circular triangle. [14].

The combined search area for any of the three $N=3$ scenarios is

$$
\begin{array}{r}
f\left(\rho_{1}, \rho_{2}, \rho_{3}, \sigma_{1,2}, \sigma_{1,3}, \sigma_{2,3}\right)= \\
\sum_{i=1}^{3} \rho_{i}^{2}\left(\pi+\sum_{j=1, j \neq i}^{3} \frac{\sin \theta_{i, j}-\theta_{i, j}}{2}\right)+2 V,
\end{array}
$$

where $\theta_{i, j}$ is defined as shown in Fig. 3. For Scenario 3, V is the area of the circular triangle [14]; $V=0$ for Scenarios 1 and 2. For the case $\rho_{1}=\rho_{2}=\rho_{3}=\rho$ and $\sigma_{1,2}=\sigma_{1,3}=$ $\sigma_{2,3}=\sigma,(31)$ becomes

$$
f(\rho, \sigma)=3 \pi \rho^{2}-2 \theta \rho^{2}+2 \sin \theta \rho^{2}+2 V,
$$

for Scenario 1 and

$$
f(\rho, \sigma)=3 \pi \rho^{2}-3 \theta \rho^{2}+3 \sin \theta \rho^{2}+2 V,
$$

for Scenarios 2 and 3 since in Scenario 1, ships 1 and 3 do not overlap in sensor coverage, thus $\theta_{1,3}=\theta_{3,1}=0$. We implemented a simulated annealing probabilistic search algorithm to find the optimal radii and spacing. [15]. The searcharea maximization algorithm for $N=3$ ships is described in Table I. Fig. 3b illustrates the optimal solution to Scenario 3 for $N=3$ when $\rho_{1}=\rho_{2}=\rho_{3}=\rho$ and $\sigma_{1,2}=\sigma_{2,3}=\sigma_{1,3}=\sigma$.

\section{OPTIMAL TARGET ASSIGNMENT}

In this section we pose and solve an optimal target assignment problem that seeks to balance the search and 


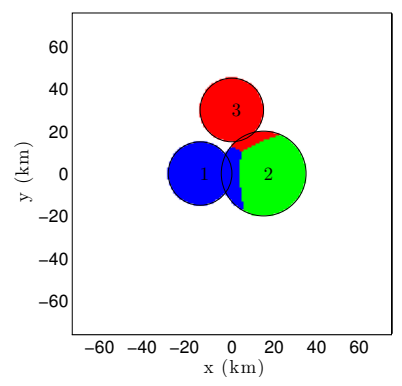

(a) Search Area Unoptimized

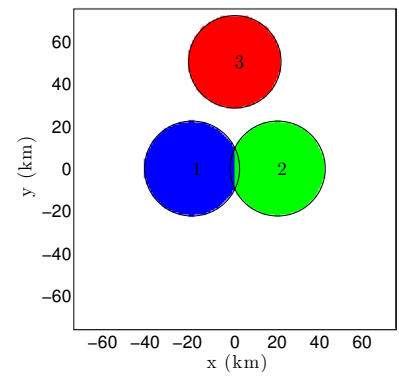

(b) Search Area Optimized

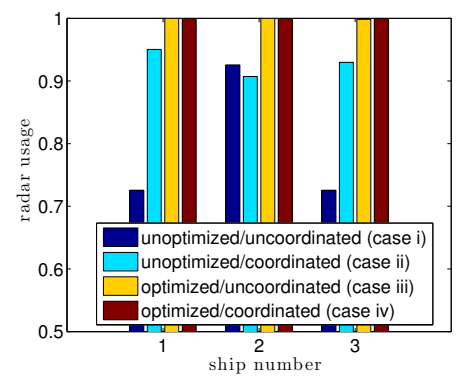

(c) Resource loading

Fig. 5: Coordinated target assignment for $N=3$ ships: Optimization increases the trackable area and balances radar tasking.

track tasking of multi-function radar systems. We show that the solution has a hyperbolic boundary determined by the search-area radii and distance to the target.

We consider four possible cases of radar performance to evaluate the target-assignment algorithm. Case (i) - Unoptimized/Uncoordinated-occurs when the radars do not optimize their combined search area and do not coordinate on the target assignment. Case (ii)Unoptimized/Coordinated-occurs when the radars do not optimize their combined search area but coordinate on the target assignment. This case could arise when the radar locations are fixed or when the radar ship platforms must remain in a particular location for other operational mission requirements. Case (iii)—Optimized/Uncoordinatedoccurs when the combined search area is optimized but the radars do not coordinate on the target assignment. This case could arise if the ship positions and radar search radii were remotely optimized prior to arriving on station. Case (iv)—Optimized/Uncoordinated—occurs when the combined search area is optimized and the radars coordinate on the target assignment.

\section{A. Single Target}

Recall the resource loading $P_{k}$ of ship $k$ defined in (3). To balance the radar tasking between all pairs of communicating ships, we seek to minimize $\left|P_{k}-P_{l}\right|$ for all pairs $k, l$, consistent with the resource reserve $\varepsilon$. We show that a balanced approach increases the trackable area and allows more targets to be tracked within a given search area.

Consider a fleet of $N$ identical ships that communicate via the undirected ship network $G_{s}=\left(\mathcal{N}, E_{S}\right)$, where $\mathcal{N}=$ $\{1, \ldots, N\}$ is the set of ship indices and $E_{s} \in \mathcal{N}^{2}$ is the set of unordered pairs of bidirectional communication links $(k, l)$. If $(k, l) \in E_{S}$, then ship $k$ may track targets within ship l's search area (as long as $P_{k} \leq 1-\varepsilon$ ).

Let $M=1$ and $j$ be the index of the tracking ship. The measure of resource balancing among all ships is

$$
C_{j}\left(G_{s}\right)=\sum_{(k, l) \in E_{s}}\left(P_{k}-P_{l}\right)^{2}
$$

TABLE II: Single Target Assignment Algorithm
Input: The ship communication network $G_{s}$, resource reserve $\varepsilon$, and the position, search radii, and search sector of each ship
At each timestep and for $m=M=1$ target:
1: Calculate $S_{k}, T_{k, m}$ and $P_{k}$ according to (1), (2), and (3) for each ship $k$.
2: Compute $C_{j}$, according to (33) where $j \in\{1,2,3\}$ denotes the tracking ship, and chose $j^{*}$ according to (34).

The optimal target assignment for $G_{s}$ that balances the radar tasking between all pairs of ships is

$$
j^{*}=\underset{j \in\{1, \ldots, N\}}{\operatorname{argmin}} C_{j}\left(G_{s}\right) .
$$

We compute the optimal target assignment for $N=3$ ships and $M=1$ target using the target-assignment optimization algorithm in Table II. Note the set of possible target positions assigned to a ship is called the track zone.

Fig. 5a shows the solution to the target-assignment problem for Case (ii); note that ship $k=1$ and ship $k=3$ are assigned tracking responsibilities inside ship $k=2$ 's search area. Fig. 5b shows the solution to the target-assignment program for Case (iv) for three ships whose combined search area is optimized using Lemma 1 and Lemma 2. Fig. 5c compares the radar usage of all four cases and verifies that the optimal target assignment balances radar tasking. In the cases where the search area and ship positions are optimized, the optimal solution also also yields an increase in the trackable area.

For Case (ii) and (iv) which coordinate their target assignment, we have the following observation for the solution of the target assignment problem described in the following Lemma: the boundaries between the set of targets positions assigned to each ship are hyperbolas.

Lemma 3: Let $\sigma_{j, m}$ and $\sigma_{k, m}$ be the distance from target $m$ to ship $j$ and ship $k$ respectively. The solution to the target assignment problem is a pair of track zones separated by a hyperbolic boundary given by the solution to

$$
\sigma_{j, m}-\sigma_{k, m}=\frac{S_{k}-S_{j}}{c} .
$$

where $S_{k}$ and $S_{j}$ are given in (2). 
TABLE III: Multi-Target Assignment Algorithm

Input: The same as the Target Assignment Algorithm in Table 2.

At each timestep and for each target assignment network

$G_{t}=\left(\mathcal{M}, E_{t}\right)$

1: Calculate $P_{k}\left(G_{t}\right)$ according to (1), (2), and (3)

2: Calculate $C\left(G_{t} ; G_{s}\right)$ according to (32)

3: Find the target assignment network $G_{t}^{*}$ that minimizes $C\left(G_{t} ; G_{s}\right)$ using simulated annealing [15].

Proof: Let $P_{k}, P_{j}$ be the resources consumed by ships $k$ and $j$ to track target $m=M=1$ as in (3). The optimal solution to the target-assignment problem switches from $k$ to $j$ as the target passes through the boundary $P_{j}=P_{k}$, i.e., when $S_{k}+T_{k}=S_{j}+T_{j}$, which is equivalent to (31).

Fig. 6a plots the hyperbolic boundaries for $N=3$ unoptimized and coordinated ships and $M=1$ target.

\section{B. Multiple Targets}

For $M \geq 2$ targets, we consider a second network, $G_{t}=$ $\left(\{\mathcal{M}, N\}, E_{t}\right)$, called the target-assignment network, where $E_{t} \in \mathcal{M} \times N$ is the set of all possible target-to-ship pairings. The measure of the resource balancing among all ships and all targets for a particular target-assignment network $G_{t}$ and ship communication network $G_{S}$ is

$$
C\left(G_{t} ; G_{s}\right)=\sum_{(k, l) \in E_{s}}\left(P_{k}\left(G_{t}\right)-P_{l}\left(G_{t}\right)\right)^{2} .
$$

Let $G_{t}^{*}=\operatorname{argmin}_{G_{t}} C\left(G_{t} ; G_{s}\right)$ be the optimal targetassignment network that balances the radar tasking across ship network $G_{s}$. We compute $G_{t}^{*}$ using the multi-target assignment algorithm in Table III. For ships that coordinate on their target assignment, we have the following result:

Lemma 4 (Multi-Target Assignment): For each pair of ships $j, k$ that coordinate on their target assignment, let $m=\left\{1, \ldots, M_{j}\right\}$ be the indices of targets tracked by ship $j$ and $l=\left\{1, \ldots, M_{k}\right\}$ be the indices of targets tracked by ship $k$, where $M_{j}+M_{k}=M-1$. The target assignment boundary for target $M$ is the solution to

$$
\begin{aligned}
& \sqrt{\left(x_{j}-x_{M}\right)^{2}+\left(y_{j}-y_{M}\right)^{2}}-\sqrt{\left(x_{k}-x_{M}\right)^{2}+\left(y_{k}-y_{M}\right)^{2}}= \\
& \sum_{l=1}^{M_{k}} \sqrt{\left(x_{k}-x_{l}\right)^{2}+\left(y_{k}-y_{l}\right)^{2}}-\sum_{m=1}^{M_{j}} \sqrt{\left(x_{j}-x_{m}\right)^{2}+\left(y_{j}-y_{m}\right)^{2}} \\
& +\frac{S_{k}-S_{j}}{c}
\end{aligned}
$$

Proof: Let $T_{j, m}$ and $T_{k, l}$ be the resources consumed by ships $j$ and $k$ to track targets $m$ and $l$. The optimal solution to the target assignment problem for the target $M$ switches from $j$ to $k$ as the target passes through the boundary $P_{j, M}=$ $P_{k, M}-\sum_{m=1}^{M_{j}} T_{j, m}+\sum_{l=1}^{M_{k}} T_{k, l}$ or equivalently

$$
\sigma_{j, M}-\sigma_{k, M}=\frac{S_{k}-S_{j}}{c}-\sum_{m=1}^{M_{j}} \sigma_{j, m}+\sum_{l=1}^{M_{k}} \sigma_{k, l},
$$

which yields (33) and completes the proof.

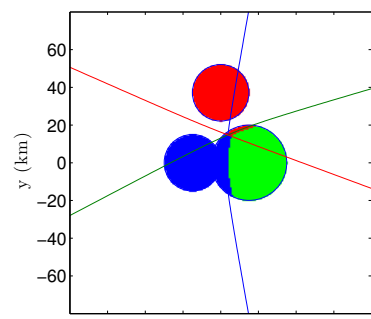

(a) $M=1$ target

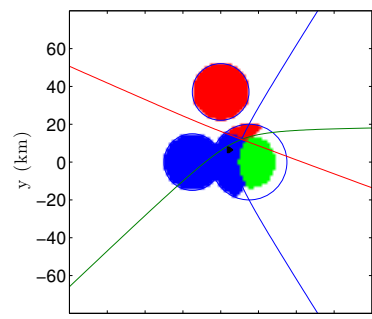

(b) $M=2$ targets
Fig. 6: Target assignment boundaries for unoptimized and coordinated ships. (Target shown as black dot.)

Fig 6 b plots the boundaries for the second of two targets when the ships are unoptimized and coordinated.

\section{CONCLUSION}

This paper provides optimization algorithms to maximize the combined search area of multiple ship-based radars that are concurrently tracking targets within the search area. It also proposes an optimal target-assignment algorithm that balances the radar tasking in both optimized and unoptimized scenarios. An area of ongoing research is to optimize the target-assignment solution for varying target characteristics and radar capabilities.

\section{REFERENCES}

[1] Johns Hopkins University, Applied Physics Laboratory, "Integrated layered defense strategy (ILDS) study on Integrated Air and Missile Defense gaps," PEO IWS and MDA, Tech. Rep., 2009.

[2] "Office of Naval Research - Radar resource manager for Integrated Air and Missile Defense,” http://tinyurl.com/7csqtvn, 2009, Future Naval Capabilities Program.

[3] S. Ghosh, J. Hansen, R. Rajkumar, and J. Lehoczky, "Integrated resource management and scheduling with multi-resource constraints," in Proc IEEE Int. Real-Time Systems Symp., 2004, pp. 12-22.

[4] P.-S. Kang and C.-G. Lee, "Coordinated search and track by multiple phased array radars," in IEEE Real-Time and Embedded Tech. and Applications Symp. IEEE Computer Society, 2004, pp. 227-235.

[5] M. P. M. Flint and E. Fernandez, "Cooperative control for multiple autonomous UAV's searching for targets," in Proc. IEEE Conf. Decision and Control, 2002, pp. 2823-2828.

[6] S. L. Smith and F. Bullo, "Monotonic target assignment for robotic networks," IEEE Trans. on Automatic Control, pp. 2042-2057, 2009.

[7] W. Ren, R. Beard, and E. Atkins, "A survey of consensus problems in multi-agent coordination," American Control Conf., pp. 1859-1864, June 2005.

[8] C. Schumacher, P. Chandler, and S. Rasmussen, "Task allocation for wide area search munitions," in Proc. American Control Conf., 2002, pp. 1917-1922.

[9] T. Li, M. Fu, L. Xie, and J. Zheng, "Distributed consensus with limited data rate," IEEE Trans. on Automatic Control, pp. 279-292, Feb. 2011.

[10] L. B. Han-Lim Choi and J. How, "Consensus-based decentralized auctions for robust task allocation," in IEEE Trans on Robotics, vol. 25, no. 4, 2009, pp. 912-926.

[11] K. J. Obermeyer, A. Ganguli, and F. Bullo, "A complete algorithm for searchlight scheduling," Int. J. Computational Geometry and Applications, pp. 101-130, 2011.

[12] D. R. Billetter, Multifunction Array Radar. Artech House, 1989.

[13] M. Skolnik, Introduction to Radar Systems. McGraw-Hill, 2001.

[14] M. Fewell, "Area of common overlap of three circles," Defence Science and Technology Organisation, Tech. Rep., Oct. 2006.

[15] D. Bertsimas and J. Tsitsiklis, "Simulated annealing," Statistical Science, vol. 8, no. 1, pp. 10-15, 1993. 